\title{
The Transmuted Geometric-Weibull distribution: Properties, Characterizations and Regression Models
}

\author{
Zohdy M. Nofal \\ Department of Statistics, Mathematics and Insurance, Benha University, Egypt \\ dr.zohdynofal@fcom.bu.edu.eg \\ Yehia M. El Gebaly \\ Department of Statistics, Mathematics and Insurance, Benha University, Egypt \\ yehia1958@yahoo.com \\ Emrah Altun \\ Department of Statistics,Hacettepe University, Turkey \\ emrahaltun@hacettepe.edu.tr \\ Morad Alizadeh \\ Department of Statistics, Persian Gulf University, Bushehr, Iran \\ moradalizadeh78@gmail.com \\ Nadeem Shafique Butt \\ Department of Family and Community Medicine, Faculy of Medicine \\ King Abdulaziz University, Kingdom of Saudi Arabia \\ nshafique@kau.edu.sa
}

\begin{abstract}
We propose a new lifetime model called the transmuted geometric-Weibull distribution. Some of its structural properties including ordinary and incomplete moments, quantile and generating functions, probability weighted moments, Rényi and q-entropies and order statistics are derived. The maximum likelihood method is discussed to estimate the model parameters by means of Monte Carlo simulation study. A new location-scale regression model is introduced based on the proposed distribution. The new distribution is applied to two real data sets to illustrate its flexibility. Empirical results indicate that proposed distribution can be alternative model to other lifetime models available in the literature for modeling real data in many areas.
\end{abstract}

Keywords: Goodness of fit, Lifetime data, Maximum likelihood, Moment, Order statistic, Regression model.

\section{Introduction}

The Weibull distribution has an undeniable popularity in probability and statistics due to its versatility of modeling real world data. Yet there are many cases where the classical Weibull distribution is unable to capture true phenomenon under study. Therefore, several of its generalizations have been proposed and studied. A generalized form of Weibull distribution is obtained by inducting one or more parameter(s) to the twoparameter Weibull distribution. It has been proven that several of these generalized distribution are more flexible and are capable of modeling real world data better than the classical Weibull distribution. A state-of-the-art survey on the class of such generalized Weibull distributions can be found in Lai et al. (2001) and Nadarajah (2009). Some 
generalization of the Weibull distribution studied in the literature includes, but are not limited to, exponentiated Weibull (Mudholkar and Srivastava, 1993; Mudholkaret al. 1995; Mudholkar, Srivastava et al. 1996), additive Weibull (Xie and Lai, 1995), Marshall-Olkin extended Weibull (Ghitany et al. 2005), beta Weibull (Famoye et al. 2005), modified Weibull (Sarhan and Zaindin, 2009), beta modified Weibull (Silva et al. 2010), transmuted Weibull (Aryal and Tsokos, 2011), extended Weibull (Xie et al. 2002), modifiedWeibull (Lai et al. 2003), KumaraswamyWeibull (Cordeiro et al. 2010), Kumaraswamy modified Weibull (Cordeiro et al. 2012), Kumaraswamy inverse Weibull (Shahbaz et al. 2012), exponentiated generalized Weibull (Cordeiro et al. 2013), McDonald modified Weibull (Merovci and Elbatal, 2013), beta inverse Weibull (Hanook et al. 2013), transmuted exponentiated generalized Weibull (Yousof et al., 2015), McDonald Weibull (Cordeiro et al. 2014), gamma Weibull (Provost et al, 2011), transmuted modified Weibull (Khan and King, 2013), beta Weibull (Lee et al. 2007), generalized transmuted Weibull (Nofal et al, 2017), transmuted additiveWeibull (Elbatal and Aryal, 2013), exponentiated generalized modified Weibull (Aryal and Elbatl, 2015), transmuted exponentiated additive Weibull (Nofal et al. 2016), Marshall Olkin additive Weibull (Afify et al. 2016), Kumaraswamy transmuted exponentiated additive Weibull (Nofal et al, 2016) distributions and the Topp-Leone Generated Weibull distribution (Aryal et al, 2016)

Let $p(t)$ be the probability density function (pdf) of a random variable $T \in a, b]$ for $-\infty<a<b<\infty$ and let $W[G(x)]$ be a function of the cumulative distribution function (cdf) of a random variable $X$ such that $W[G(x)]$ satisfies the following conditions:

$\left(\begin{array}{ll}(i) & W[G(x)] \in a, b], \\ \text { (ii) } & W[G(x)] \text { isdifferentiableandmonotonicallynon }- \text { decreasing, and } \\ \text { (iii) } & W[G(x)] \rightarrow a \text { as } x \rightarrow-\infty \text { and } W[G(x)] \rightarrow b \text { as } x \rightarrow \infty .\end{array}\right.$

Recently, Alzaatreh et al. (2013) defined the $T$-X family of distributions by

$$
F(x)=\int_{a}^{W[G(x)]} p(t) d t
$$

where $W[G(x)]$ satisfies conditions (1). The pdf corresponding to (2) is given by

$$
f(x)=\left\{\frac{d}{d x} W[G(x)]\right\} p\{W[G(x)]\} .
$$

According to Afify et al. (2016) the cdf of the TG-G family is given by

$$
\begin{aligned}
F_{T G-G}(x)=\int_{0}^{\frac{\theta G(x ; \phi)}{1+(\theta-1) G(x ; \phi)}}(1+\lambda-2 \lambda t) d t \\
\quad=\frac{\theta G(x ; \phi)}{1+(\theta-1) G(x ; \phi)}\left\{1+\frac{\lambda \bar{G}(x ; \phi)}{1+(\theta-1) G(x ; \phi)}\right\},
\end{aligned}
$$

where $G(x ; \phi)$ and $\theta>0,|\lambda| \leq 1$ are two additional shape parameters. The TG-G is a wider class of continuous distributions. It includes the transmuted-G family of distributions and geometric-G. Concider the cdf of the Weibull (W) distribution, $G(x ; \alpha, \beta)=1-\mathrm{e}^{-(\alpha x)^{\beta}}$, Then using $F_{T G-G}(x)$ we get

$$
F(x)=\frac{\theta\left(1-\mathrm{e}^{-(\alpha x)^{\beta}}\right)}{1+(\theta-1)\left(1-\mathrm{e}^{-(\alpha x)^{\beta}}\right)}\left\{1+\frac{\lambda \mathrm{e}^{-(\alpha x)^{\beta}}}{1+(\theta-1)\left(1-\mathrm{e}^{-(\alpha x)^{\beta}}\right)}\right\}
$$


The pdf corresponding of (4) is given by

$f(x)=\frac{\theta \beta \alpha^{\beta} x^{\beta-1}}{\mathrm{e}^{(\alpha x)^{\beta}}}\left[1+(\theta-1)\left(1-\mathrm{e}^{-(\alpha x)^{\beta}}\right)\right]^{-2}\left[1+\lambda-\frac{2 \lambda \theta\left(1-\mathrm{e}^{-(\alpha x)^{\beta}}\right)}{1+(\theta-1)\left(1-\mathrm{e}^{-(\alpha x)^{\beta}}\right)}\right]$.

For $\lambda=0$ we obtain geometric-W (GW) distribution. We denote by $X \sim \mathrm{TG}-\mathrm{W}(\lambda, \theta, \alpha, \beta)$ a random variable having density function (5).

The rest of the paper is organized as follows: In Section 2, some mathematical properties of the TGW are obtained such as mixture representation, quantile function, moments, order statistics and reliability estimation. Section 4 is devoted to characterizations of the proposed distribution and in Section 5 estimation of model parameters by the maximum likelihood method is presented. In Section 5, brief Monte-Carlo simulation study is performed to estimate model parameters with maximum likelihood estimators (MLE). The log-transmuted geometric-Weibull regression model is defined in Section 6. Section 7 is devoted to applications to illustrate the flexibility of the proposed distribution in many fields such as univariate data fitting and survival analysis. Finally, some concluding remarks are given in Section 8.

\section{Mathematical Properties}

In this section some mathematical properties of the TWG distribution is discussed.

\subsection{Survival and Hazard Functions}

Central role is played in the reliability theory by the quotient of the pdf and survival function. We obtain the survival function corresponding to (4) as

$$
S(x)=1-\frac{\theta\left(1-\mathrm{e}^{-(\alpha x)^{\beta}}\right)}{1+(\theta-1)\left(1-\mathrm{e}^{-(\alpha x)^{\beta}}\right)}\left\{1+\frac{\lambda \mathrm{e}^{-(\alpha x)^{\beta}}}{1+(\theta-1)\left(1-\mathrm{e}^{-(\alpha x)^{\beta}}\right)}\right\}
$$

In reliability studies, the hazard rate function (hrf) is an important characteristic and fundamental to the design of safe systems in a wide variety of applications. Therefore, we discuss these properties of the TGW distribution. The hrf of X takes the form

$$
\begin{aligned}
& h(x)=\frac{\theta \beta \alpha^{\beta} x^{\beta-1}}{\mathrm{e}^{(\alpha x)^{\beta}}}\left[1+(\theta-1)\left(1-\mathrm{e}^{-(\alpha x)^{\beta}}\right)\right]^{-2}\left[1+\lambda-\frac{2 \lambda \theta\left(1-\mathrm{e}^{-(\alpha x)^{\beta}}\right)}{1+(\theta-1)\left(1-\mathrm{e}^{-(\alpha x)^{\beta}}\right)}\right] \\
& \quad \times\left\{1-\frac{\theta\left(1-\mathrm{e}^{-(\alpha x)^{\beta}}\right)}{1+(\theta-1)\left(1-\mathrm{e}^{-(\alpha x)^{\beta}}\right)}\left\{1+\frac{\lambda \mathrm{e}^{-(\alpha x)^{\beta}}}{1+(\theta-1)\left(1-\mathrm{e}^{-(\alpha x)^{\beta}}\right)}\right\}\right\}^{-1} .
\end{aligned}
$$

In Figure 1, we display some plots of the pdf and hrf of the TGW distribution for selected parameter values. Figure 1 reveals that the TGW density generate various shapes such as right-skewed, reversed-J, and unimodal. Figure 1 also shows that the TGW distribution can produce hazard rate shapes such as increasing, decreasing, and reversed-J. This fact implies that the TGW distribution can be very useful for fitting data sets with various shapes. 

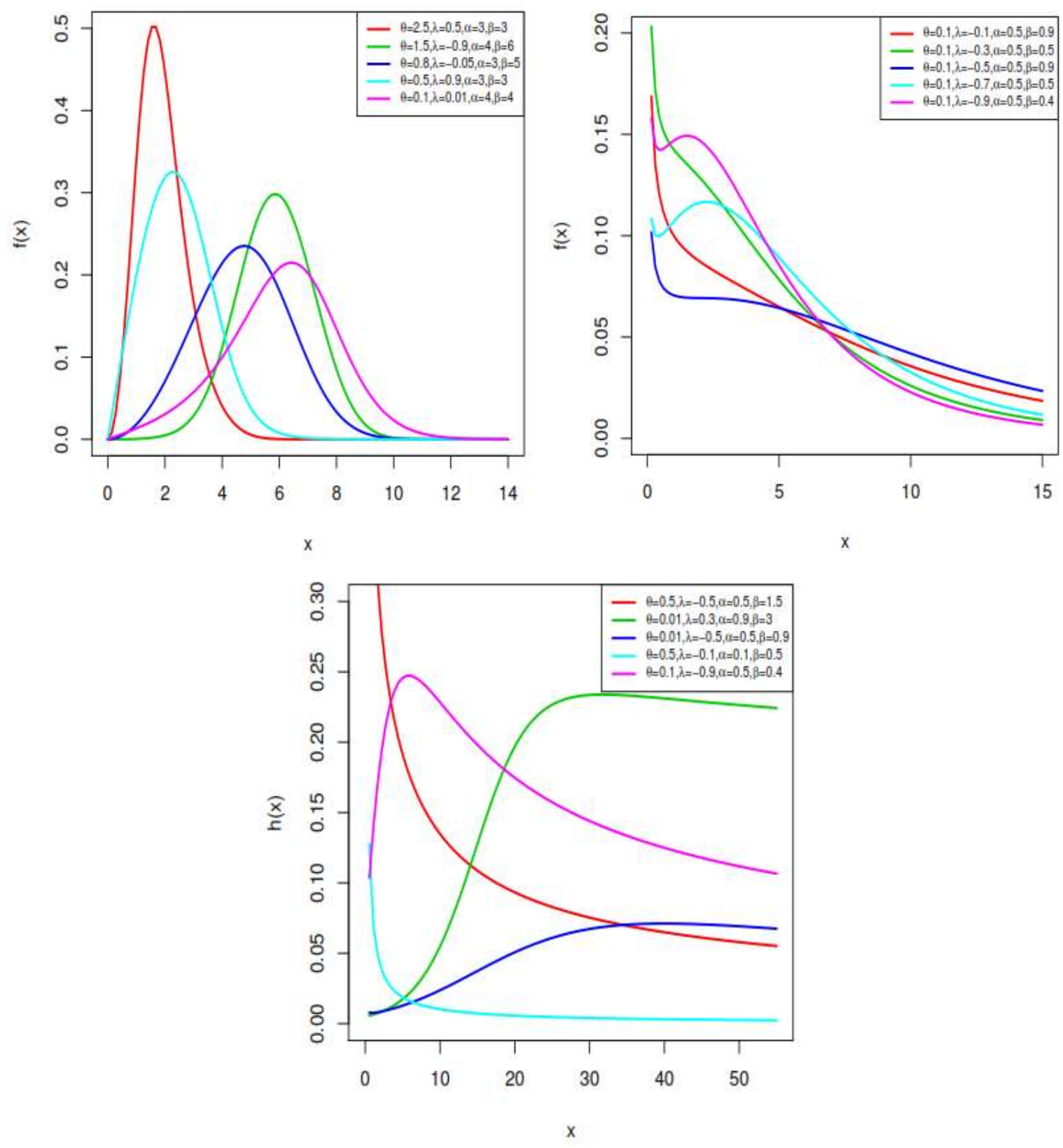

Figure 1. Plots of pdf and hrf of the TGW distribution for the selected parameter values.

\subsection{Asymptotics}

Proposition 2.1 The asymptotics of TGW distribution from cdf, pdf and hrf as $x \rightarrow 0$ are given by

$$
\begin{aligned}
& F(x): \theta(1+\lambda)(\alpha x)^{\beta}, \\
& f(x): \theta \beta(1+\lambda) \alpha^{\beta} x^{\beta-1}, \\
& h(x): \theta \beta(1+\lambda) \alpha^{\beta} x^{\beta-1} .
\end{aligned}
$$


Proposition 2.2 The asymptotics of TGW distribution from cdf, pdf and hrf as $x \rightarrow \infty$ are given by

$$
\begin{aligned}
& 1-F(x): \mathrm{e}^{-(\alpha x)^{\beta}} \\
& f(x): \beta \alpha^{\beta} x^{\beta-1} \mathrm{e}^{-(\alpha x)^{\beta}} \\
& h(x): \beta \alpha^{\beta} x^{\beta-1} .
\end{aligned}
$$

These equations show the effect of parameters on tails of TGW distribution.

\subsection{Mixture Representation}

In this section, we provide a very useful representation for the TG-W density. The pdf in (5) can be rewritten as

$$
f(x)=\frac{\theta(1+\lambda) \beta \alpha^{\beta} x^{\beta-1} \mathrm{e}^{-(\alpha x)^{\beta}}}{\left[1+(\theta-1)\left(1-\mathrm{e}^{-(\alpha x)^{\beta}}\right)\right]^{2}}-\frac{2 \lambda \theta^{2} \beta \alpha^{\beta} x^{\beta-1} \mathrm{e}^{-(\alpha x)^{\beta}}\left(1-\mathrm{e}^{-(\alpha x)^{\beta}}\right)}{\left[1+(\theta-1)\left(1-\mathrm{e}^{-(\alpha x)^{\beta}}\right)\right]^{3}}
$$

Then, the pdf in (6) can be rewritten as

$$
\begin{gathered}
f(x)=(1+\lambda) \theta \beta \alpha^{\beta} x^{\beta-1} \mathrm{e}^{-(\alpha x)^{\beta}} \sum_{k=0}^{\infty}(\theta-1)^{k}\left(\begin{array}{l}
-2 \\
k
\end{array}\right)\left(1-\mathrm{e}^{-(\alpha x)^{\beta}}\right)^{k} \\
-2 \lambda \theta^{2} \beta \alpha^{\beta} x^{\beta-1} \mathrm{e}^{-(\alpha x)^{\beta}} \sum_{k=0}^{\infty}(\theta-1)^{k}\left(\begin{array}{l}
-3 \\
k
\end{array}\right)\left(1-\mathrm{e}^{-(\alpha x)^{\beta}}\right)^{k+1} .
\end{gathered}
$$

the pdf (7) can be expressed as a mixture of exp-W density

$$
f(x)=\sum_{k=0}^{\infty}\left[a_{k} \pi_{k+1}(x)-b_{k} \pi_{k+2}(x)\right] .
$$

But

where

$$
\left(\begin{array}{l}
-2 \\
k
\end{array}\right)\left(=(-1)^{k}(k+1) \quad \text { and } \quad\left(\begin{array}{l}
-3 \\
k
\end{array}\right)=\frac{(-1)^{k}(k+1)(k+2)}{2},\right.
$$

$$
\pi_{\delta}(x)=\delta \beta \alpha^{\beta} x^{\beta-1} \mathrm{e}^{-(\alpha x)^{\beta}}\left(1-\mathrm{e}^{-(\alpha x)^{\beta}}\right)^{\delta-1}
$$

is the exp-G pdf with power parameter $\delta>0$,

$$
a_{k}=\theta(1+\lambda)(1-\theta)^{k} \quad \text { and } \quad b_{k}=\lambda \theta^{2}(k+1)(1-\theta)^{k}
$$

Thus, several mathematical properties of the TG-W density can be obtained simply from those properties of the exp-W density. Equation (8) is the main result of this section.

The cdf of the TG-W distribution can also be expressed as a mixture of exp-W densities. By integrating (8), we obtain the same mixture representation

$$
F(x)=\sum_{k=0}^{\infty}\left[a_{k} \Pi_{k+1}(x)-b_{k} \Pi_{k+2}(x)\right],
$$

where $\Pi_{\delta}(x)=\left(1-\mathrm{e}^{-(\alpha x)^{\beta}}\right)^{\delta}$ is the cdf of the exp-W density with power parameter $\delta$. 


\subsection{Quantile Function}

The quantile function (qf ) of $X$, where $X$ :TG-W $(\lambda, \theta, \alpha, \beta)$, is obtained by inverting (3) to obtain $Q(u)=F^{-1}(u)$ as

$Q(u)=\frac{1}{\alpha}\left[\ln \left[\frac{2 u(1-\theta)^{2}+2 \theta(1+\lambda)-2 \theta^{2}}{2 u \theta(1-\theta)+\theta(1+\lambda)-2 \theta^{2}+\theta \sqrt{(1+\lambda)^{2}-4 u \lambda}}\right]\right]^{\frac{1}{\beta}}$, for $\lambda \neq 0, u \in(0,1)$.

For $\lambda=0$, we have

$Q(u)=\frac{1}{\alpha}\left[\ln \left[\frac{u(1-\theta)^{2}+\theta-\theta^{2}}{u \theta(1-\theta)+\theta-\theta^{2}}\right]\right]^{\frac{1}{\beta}}$

Simulating the TG-W random variable is straightforward. If $U$ is a uniform variate on the unit interval $(0,1)$, then the random variable $X=Q(U)$ follows 6 .

The effects of the shape parameters on the skewness and kurtosis can be based on quantile measures. We obtain skewness and kurtosis measures using the qf. The Bowley's skewness measure is given by

$$
\text { Skewness }=\frac{Q(1 / 4)+Q(3 / 4)-2 Q(1 / 2)}{Q(3 / 4)-Q(1 / 4)}
$$

and the Moors's kurtosis measure is

$$
\text { Kurtosis }=\frac{Q(7 / 8)-Q(5 / 8)+Q(3 / 8)-Q(1 / 8)}{Q(6 / 8)-Q(2 / 8)} \text {. }
$$

These measures enjoy the advantage of having less sensitivity to outliers. Moreover, they do exist for distribution without moments. Both measures equal zero for the normal distribution. Plots of skewness and kurtosis of the TGW distribution are presented in Figure 2. These plots indicate that both measures depend very much on the shape parameters. Therefore TGW distribution can model various data types in terms of skewness and kurtosis.

\section{Skewness}

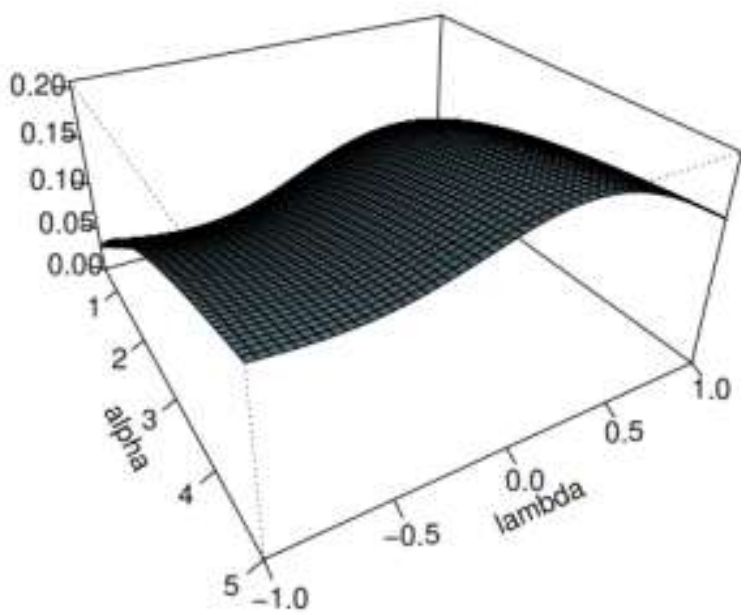

Kurtosis

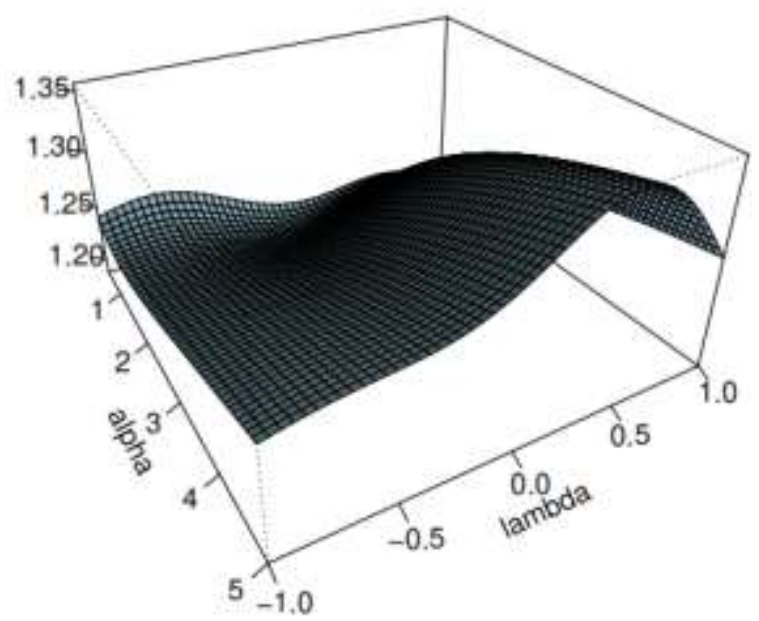

Figure 2. Plots of skewness and kurtosis of TGW distribution for $\alpha=2$ and $\beta=2$. 


\subsection{Moments and Generating Function}

The $r$ th moment of $X$, say $\mu_{r}^{\prime}$, follows from (9) as

Where

$$
\mu_{r}^{\prime}=E\left(X^{r}\right)=\sum_{k=0}^{\infty}\left\{a_{k} E\left(Y_{k+1}^{r}\right)-b_{k} E\left(Y_{k+2}^{r}\right)\right\}=\sum_{k, j=0}^{\infty} \boldsymbol{\omega}_{k, j} \Gamma\left(1+\frac{r}{\beta}\right) .
$$

$$
\boldsymbol{\omega}_{k, j}=\frac{\alpha^{-r}(-1)^{j}}{j !(j+1)^{(r+\beta) / \beta}}\left\{\frac{a_{k} \Gamma(k+2)}{\Gamma(k+1-j)}-\frac{b_{k} \Gamma(k+3)}{\Gamma(k+2-j)}\right\}
$$

Henceforth, $Y_{k}$ denotes the exp-G distribution with power parameter $k$. The $n$th central moment of $X$, say $M_{n}$, is given by

$$
\begin{gathered}
M_{n}=E\left(X-\mu_{1}^{\prime}\right)^{n}=\sum_{r=0}^{n}\left(\begin{array}{l}
n \\
r
\end{array}\right)\left(-\mu_{1}^{\prime}\right)^{n-r} E\left(X^{r}\right) \\
=\sum_{r=0}^{n} \sum_{k, j=0}^{\infty}(-1)^{n-r}\left(\begin{array}{l}
n \\
r
\end{array}\right) \mu_{r}^{(n-r)} \boldsymbol{\omega}_{k, j} \Gamma\left(1+\frac{r}{\beta}\right) .
\end{gathered}
$$

The cumulants $\left(\kappa_{n}\right)$ of $X$ follow recursively from

$$
\kappa_{n}=\mu_{n}^{\prime}-\sum_{r=0}^{n-1}\left(\begin{array}{l}
n-1 \\
r-1
\end{array}\right) \kappa_{r} \mu_{n-r}^{\prime}
$$

where $\kappa_{1}=\mu_{1}^{\prime}, \kappa_{2}=\mu_{2}^{\prime}-\mu_{1}^{\prime 2}, \kappa_{3}=\mu_{3}^{\prime}-3 \mu_{2}^{\prime} \mu_{1}^{\prime}+\mu_{1}^{\prime 3}$, etc. The skewness $\gamma_{1}=\kappa_{3} / \kappa_{2}^{3 / 2}$ and kurtosis $\gamma_{2}=\kappa_{4} / \kappa_{2}^{2}$ are obtained from the third and fourth standardized cumulants. The $n$th descending factorial moment of $X$ (for $n=1,2, \ldots$ ) is

$$
\mu_{(n)}^{\prime}=E\left[X^{(n)}\right]=E[X(X-1) \times \ldots \times(X-n+1)]=\sum_{k=0}^{n} s(n, k) \mu_{k}^{\prime},
$$

where $s(n, k)=(k !)^{-1}\left[d^{k} k^{(n)} / d x^{k}\right]_{x=0}$ is the Stirling number of the first kind. The mgf $M_{X}(t)=E\left(e^{t X}\right)$ of $X$ can be derived from equation (8) as

$$
M_{X}(t)=\sum_{k=0}^{\infty}\left[a_{k} M_{k+1}(t)-b_{k} M_{k+2}(t)\right]
$$

where $M_{k}(t)$ is the mgf of $Y_{k}$. Hence, $M_{X}(t)$ can be determined from the exp-G generating function. Then

$$
M_{X}(t)=\sum_{k, j, r=0}^{\infty} m_{k, j, r} \Gamma\left(1+\frac{r}{\beta}\right),
$$

where $m_{k, j, r}=\boldsymbol{\omega}_{k, j} \frac{t^{r}}{r !}$.

\subsection{Incomplete Moments and Mean Deviations}

The main applications of the first incomplete moment refer to the mean deviations and the Bonferroni and Lorenz curves. These curves are very useful in economics, reliability, 
demography, insurance and medicine. The $s$ th incomplete moment, say $\varphi_{r}(t)$, of $X$ can be expressed from (8) as

$$
\begin{aligned}
& \varphi_{r}(t)=\int_{-\infty}^{t} x^{r} f(x) d x=\sum_{k=0}^{\infty}\left[a_{k} \int_{-\infty}^{t} x^{r} \pi_{k+1}(x) d x-b_{k} \int_{-\infty}^{t} x^{r} \pi_{k+2}(x) d x\right] . \\
& =\sum_{k, j=0}^{\infty} \boldsymbol{\omega}_{k, j} \gamma\left(1+\frac{r}{\beta},\left(\frac{\alpha}{t}\right)^{\beta}\right) .
\end{aligned}
$$

The mean deviations about the mean $\left[\theta_{1}=E\left(\left|X-\mu_{1}^{\prime}\right|\right)\right]$ and about the median $\left[\theta_{2}=\right.$ $E(|X-M|)]$ of $X$ are given by $\theta_{1}=2 \mu_{1}^{\prime} F\left(\mu_{1}^{\prime}\right)-2 \varphi_{1}\left(\mu_{1}^{\prime}\right)$ and $\theta_{2}=\mu_{1}^{\prime}-2 \varphi_{1}(M)$, respectively, where $\mu_{1}^{\prime}=E(X), M=\operatorname{Median}(X)=Q(0.5)$ is the median, $F\left(\mu_{1}^{\prime}\right)$ is easily calculated from (4) and $\varphi_{1}(t)$ is the first incomplete moment given by (9) with $s=$ 1.

The general equation for $\varphi_{1}(t)$ can be derived from (9) as

where

$$
\varphi_{1}(t)=\sum_{k=0}^{\infty}\left[a_{k} J_{k+1}(x)-b_{k} J_{k+2}(x)\right]=\sum_{k, j=0}^{\infty} \boldsymbol{\omega}_{k, j}^{*} \gamma\left(1+\frac{1}{\beta},\left(\frac{\alpha}{t}\right)^{\beta}\right),
$$

$$
\boldsymbol{\omega}_{k, j}^{*}=\frac{\alpha^{-1}(-1)^{j}}{j !(j+1)^{(1+\beta) / \beta}}\left\{\frac{a_{k} \Gamma(k+2)}{\Gamma(k+1-j)}-\frac{b_{k} \Gamma(k+3)}{\Gamma(k+2-j)}\right\}
$$

These equations for $\varphi_{1}(t)$ can be applied to construct Bonferroni and Lorenz curves defined for a given probability $\pi$ by $B(\pi)=\varphi_{1}(q) /\left(\pi \mu_{1}^{\prime}\right)$ and $L(\pi)=\varphi_{1}(q) / \mu_{1}^{\prime}$, respectively, where $\mu_{1}^{\prime}=E(X)$ and $q=Q(\pi)$ is the qf of $X$ at $\pi$.

\subsection{Order Statistics}

Order statistics make their appearance in many areas of statistical theory and practice. Let $X_{1}, \ldots, X_{n}$ be a random sample from the TG-W distributions. The pdf of $i$ th order statistic, say $X_{i: n}$, can be written as

$$
f_{i: n}(x)=\frac{f(x)}{\mathrm{B}(i, n-i+1)} \sum_{j=0}^{n-i}(-1)^{j}\left(\begin{array}{l}
n-i \\
j
\end{array}\right) F(x)^{j+i-1} .
$$

Then

$$
F(x)^{j+i-1}=\sum_{w=0}^{\infty}(j+i-1)_{w} \frac{\lambda^{w} \theta^{j+i-1} \mathrm{e}^{-w(\alpha x)^{\beta}}\left(1-\mathrm{e}^{-(\alpha x)^{\beta}}\right)^{j+i-1}}{w !\left[1+(\theta-1)\left(1-\mathrm{e}^{-(\alpha x)^{\beta}}\right)\right]^{j+i+w-1}} .
$$

Using (5) and (11) we get

$$
\begin{aligned}
& f(x) F(x)^{j+i-1}=\sum_{w=0}^{\infty} \frac{(1+\lambda) \lambda^{w} \theta^{j+i} \beta \alpha^{\beta}(j+i-1)_{w} x^{\beta-1}\left(1-\mathrm{e}^{-(\alpha x)^{\beta}}\right)^{j+i-1}}{w ! \mathrm{e}^{(w+1)(\alpha x)^{\beta}}\left[1+(\theta-1)\left(1-\mathrm{e}^{-(\alpha x)^{\beta}}\right)\right]^{j+i+w+1}} \\
& -\sum_{w=0}^{\infty} \frac{2 \lambda^{w+1} \theta^{j+i+1} \beta \alpha^{\beta}(j+i-1)_{w} x^{\beta-1}\left(1-\mathrm{e}^{-(\alpha x)^{\beta}}\right)^{j+i}}{w ! \mathrm{e}^{(w+1)(\alpha x)^{\beta}}\left[1+(\theta-1)\left(1-\mathrm{e}^{-(\alpha x)^{\beta}}\right)\right]^{j+i+w+2}}
\end{aligned}
$$


Then

$$
f(x) F(x)^{j+i-1}=\sum_{k=0}^{\infty}\left[\Upsilon_{k} \pi_{k+j+i+m}(x)-\Psi_{k} \pi_{k+j+i+m+1}(x)\right] .
$$

Substituting (12) in Equation (10), the pdf of $X_{i: n}$ can be expressed as

$$
f_{i: n}(x)=\frac{\sum_{j=0}^{n-i}(-1)^{j}\left(\begin{array}{l}
n-i \\
j
\end{array}\right)}{\mathrm{B}(i, n-i+1)} \sum_{k=0}^{\infty}\left[\Upsilon_{k} \pi_{k+j+i+m}(x)-\Psi_{k} \pi_{k+j+i+m+1}(x)\right],
$$

where

$$
\begin{gathered}
\Upsilon_{k}=\sum_{m, w=0}^{\infty}(j+i-1)_{w} \frac{(-1)^{k}(1+\lambda) \lambda^{\mathrm{w}} \theta^{j+i}(1-\theta)^{m} \Gamma(w+1) \Gamma(j+i+w+m+1)}{w ! m ! k ! \Gamma(w-k+1) \Gamma(j+i+w+2)[k+j+i+m+1]} \\
\Psi_{k}=\sum_{m, w=0}^{\infty}(j+i-1)_{w} \frac{(-1)^{k} 2 \lambda^{w+1} \theta^{j+i+1}(1-\theta)^{m} \Gamma(w+1) \Gamma(j+i+w+m+2)}{w ! m ! k ! \Gamma(w-k+1) \Gamma(j+i+w+1)[k+j+i+m]}
\end{gathered}
$$

and $\pi_{k}(x)$ is the exp-W density with power parameter $k$. Then, the density function of the TG-G order statistics is a mixture of exp-G densities. Based on the last equation, we note that the properties of $X_{i: n}$ follow from those properties of $Y_{a+k}$. For example, the moments of $X_{i: n}$ can be expressed as

where

$$
\begin{aligned}
& E\left(X_{i: n}^{q}\right)=\frac{\sum_{j=0}^{n-i}(-1)^{j}\left(\begin{array}{l}
n-i \\
j
\end{array}\right)}{\mathrm{B}(i, n-i+1)} \sum_{k=0}^{\infty}\left[\Upsilon_{k} E\left(Y_{k+j+i+m}^{q}\right)-\Psi_{k} E\left(Y_{K+j+i+m+1}^{q}\right)\right] \\
& =\frac{\sum_{j=0}^{n-i}(-1)^{j}\left(\begin{array}{l}
n-i \\
j
\end{array}\right)}{\mathrm{B}(i, n-i+1)} \sum_{k, h=0}^{\infty} S_{k, h} \Gamma\left(1+\frac{q}{\beta}\right) .
\end{aligned}
$$

$$
s_{k, h}=\frac{\alpha^{-q}(-1)^{h}}{h !(h+1)^{(q+\beta) / \beta}}\left[\frac{\Gamma(K+j+i+m+1) \Upsilon_{k}}{\Gamma(K+j+i+m-h)}-\frac{\Gamma(K+j+i+m+2) \Psi_{k}}{\Gamma(K+j+i+m+1-h)}\right] .
$$

The L-moments are analogous to the ordinary moments but can be estimated by linear combinations of order statistics. They exist whenever the mean of the distribution exists, even though some higher moments may not exist, and are relatively robust to the effects of outliers. Based upon the moments in equation (13), we can derive explicit expressions for the L-moments of $X$ as infinite weighted linear combinations of the means of suitable TG-W order statistics. They are linear functions of expected order statistics defined by

$$
\lambda_{r}=\frac{1}{r} \sum_{d=0}^{r-1}(-1)^{d}\left(\begin{array}{l}
r-1 \\
d
\end{array}\right) E\left(X_{r-d: r}\right), r \geq 1
$$

\subsection{Probability Weighted Moments}

Generally, the PWM method can be used for estimating parameters of a distribution whose inverse form cannot be expressed explicitly. The PWMs are expectations of certain functions of a random variable and they can be defined for any random variable whose ordinary moments exist. They have low variance and no severe bias and can 
compare favorably with estimators obtained by the maximum likelihood method. The $(s, r)$ th PWM of $X$ following the TG-W distribution, say $\rho_{s, r}$, is formally defined by

$$
\begin{aligned}
& \rho_{s, r}=E\left\{X^{s} F(X)^{r}\right\}=\int_{-\infty}^{\infty} x^{s} F(X)^{r} f(x) d x . \\
& F(X)^{r}=\frac{\theta^{r}\left(1-\mathrm{e}^{-(\alpha x)^{\beta}}\right)^{r}}{\left[1+(\theta-1)\left(1-\mathrm{e}^{-(\alpha x)^{\beta}}\right)\right]^{r}}\left[1+\frac{\lambda\left[1-\left(1-\mathrm{e}^{-(\alpha x)^{\beta}}\right)\right]}{1+(\theta-1)\left(1-\mathrm{e}^{-(\alpha x)^{\beta}}\right)}\right]^{r} \\
& \quad=\sum_{w=0}^{\infty}(r)_{w} \frac{\lambda^{w} \theta^{r}\left[1-\left(1-\mathrm{e}^{-(\alpha x)^{\beta}}\right)\right]^{w}\left(1-\mathrm{e}^{-(\alpha x)^{\beta}}\right)^{r}}{w !\left[1+(\theta-1)\left(1-\mathrm{e}^{-(\alpha x)^{\beta}}\right)\right]^{r+w}} .
\end{aligned}
$$

From Equation (5) and the last equation, we can write

where

$$
f(x) F(x)^{r}=f(x) F(x)^{j+i-1}=\sum_{k=0}^{\infty}\left[\Upsilon_{k}^{*} \pi_{k+r+m+1}(x)-\Psi_{k}^{*} \pi_{k+r+m+2}(x)\right],
$$

and

$$
\Upsilon_{k}^{*}=\sum_{m, w=0}^{\infty}(r)_{w} \frac{(-1)^{k}(1+\lambda) \lambda^{w} \theta^{r+1}(1-\theta)^{m} \Gamma(w+1) \Gamma(r+w+m+2)}{w ! m ! k ! \Gamma(w-k+1) \Gamma(r+w+2)[k+r+m+1]}
$$

$$
\Psi_{k}^{*}=\sum_{m, w=0}^{\infty}(r)_{w} \frac{(-1)^{k} 2 \lambda^{w+1} \theta^{r+2}(1-\theta)^{m} \Gamma(w+1) \Gamma(r+w+m+3)}{w ! m ! k ! \Gamma(w-k+1) \Gamma(r+w+3)[k+r+m+2]}
$$

Finally, the $(s, r)$ th PWM of $X$ can be obtained from an infinite linear combination of exp-W moments given by

where

$$
\rho_{s, r}=\sum_{k, j=0}^{\infty} p_{k, j} \Gamma\left(1+\frac{s}{\beta}\right)
$$

$$
p_{k, j}=\frac{(-1)^{j} \alpha^{-s}}{j !(j+1)^{(s+\beta) / \beta}}\left[\frac{\Gamma(k+r+m+2) \Upsilon_{k}^{*}}{\Gamma(k+r+m+1-j)}-\frac{\Gamma(k+r+m+3) \Psi_{k}^{*}}{\Gamma(k+r+m+2-j)}\right] .
$$

\subsection{Reliability estimation}

The stress-strength model is the most widely approach used for reliability estimation. This model is used in many applications of physics and engineering such as strength failure and system collapse. In stress-strength modeling, $\mathbf{R}=\operatorname{Pr}\left(X_{2}<X_{1}\right)$ is a measure of reliability of the system when it is subjected to random stress $X_{2}$ and has strength $X_{1}$. The system fails if and only if the applied stress is greater than its strength and the component will function satisfactorily whenever $X_{1}>X_{2}$. $\mathbf{R}$ can be considered as a measure of system performance and naturally arise in electrical and electronic systems. Other interpretation can be that, the reliability, say $\mathbf{R}$, of the system is the probability that the system is strong enough to overcome the stress imposed on it. Let $X_{1}$ and $X_{2}$ be two independent random variables with $\mathrm{TG}-\mathrm{W}\left(\lambda_{1}, \theta_{1}, \alpha, \beta\right)$ and $\mathrm{TG}-\mathrm{W}\left(\lambda_{2}, \theta_{2}, \alpha, \beta\right)$ distributions. Then, the reliability is defined by

$$
\mathbf{R}=\int_{0}^{\infty} f_{1}\left(x ; \lambda_{1}, \theta_{1}, \alpha, \beta\right) F_{2}\left(x ; \lambda_{2}, \theta_{2}, \alpha, \beta\right) d x=\sum_{k, j=0}^{\infty}\left\{a_{k, j}-b_{k, j}-c_{k, j}+d_{k, j}\right\},
$$


where

and

$$
\begin{aligned}
& a_{k, j}=\frac{\left(1+\lambda_{1}\right)\left(1+\lambda_{2}\right)\left(1-\theta_{1}\right)^{k}\left(1-\theta_{2}\right)^{j}(k+1)}{(k+j+2)\left(\theta_{1} \theta_{2}\right)^{-1}} \\
& b_{k, j}=\frac{\left(1+\lambda_{1}\right)\left(1-\theta_{1}\right)^{k}\left(1-\theta_{2}\right)^{j}(k+1)(j+1)}{(k+j+3)\left(\lambda_{2} \theta_{1} \theta_{2}^{2}\right)^{-1}} \\
& c_{k, j}=\sum_{k=0}^{\infty} \frac{\left(1+\lambda_{2}\right)\left(1-\theta_{1}\right)^{k}\left(1-\theta_{2}\right)^{j}(k+2)(k+1)}{(k+j+3)\left(\lambda_{1} \theta_{1}^{2} \theta_{2}\right)^{-1}}
\end{aligned}
$$

$$
d_{k, j}=\sum_{k, j=0}^{\infty} \frac{\left(1-\theta_{1}\right)^{k}\left(1-\theta_{2}\right)^{j}(k+2) \lambda_{2}(j+1)(k+1)}{(k+j+4)\left(\lambda_{1} \lambda_{2} \theta_{1}^{2} \theta_{2}^{2}\right)^{-1}}
$$

\section{Characterizations}

Here, we provide characterizations of the GT-W distribution in terms of two truncated moments. This characterization result is based on a theorem (see Theorem 1 below) due to Glänzel (1987). The proof of Theorem 1 is given in Glänzel (1990). This result holds also when the interval $H$ is not closed. Moreover, as mentioned above, it could be also applied when the cdf $F$ does not have a closed form. Glänzel (1990) proved that this characterization is stable in the sense of weak convergence.

Theorem 1. Let $(\Omega, p)$ be a given probability space and let $H=[a, b]$ be an interval for some $a<b(a=-\infty, b=\infty$ mightaswellbeallowed). Let $H: \Omega \rightarrow H$ be acontinuous random variable with cdf $F$ and let $g$ and $h$ be two real functions defined on $H$ such that

$$
E(g(x) \mid X \geq x)=E(h(x) \mid X \geq x) \eta(x), x \in H,
$$

is defined with a real function $h$. Assume that $g, h \in C^{1}(H), \eta \in C^{2}(H)$ and $F$ is twice continuously differentiable and strictly monotone function on the set $H$. Finally, assume that the equation $h \eta=g$ has no real solution in the interior of $H$. Then $F$ is uniquely determined by the functions $g, h$ and $\eta$, particularly

$$
F(x)=\int_{a}^{x} C\left|\frac{\eta^{\prime}(u)}{\eta(u) h(u)-g(u)}\right| \exp (-s(u)) d u,
$$

where the function $s$ is a solution of the differential equation $s^{\prime}=\eta^{\prime} h /(\eta h-g)$ and $C$ is the normalization constant, such that ${ }_{H} d F=1$.

\section{Proposition 1.}

Let $X: \Omega \rightarrow(0, \infty)$ be a continuous random variable and let

and

$$
h(x)=\left[1+\lambda-\frac{2 \lambda \theta\left(1-e^{-(\alpha x)^{\beta}}\right)}{1+(\theta-1)\left(1-e^{-(\alpha x)^{\beta}}\right)}\right]^{-1}
$$

$$
g(x)=h(x)\left[1+(\theta-1)\left(1-e^{-(\alpha x)^{\beta}}\right)\right]^{-1}
$$


The random variable $X$ belongs to GT-W distribution (5) if and only if the function $\eta$ defined in Theorem 1 has the formand

Proof.

$$
\eta(x)=\frac{1}{2 \theta}\left[\frac{2 \theta-(\theta-1) e^{-(\alpha x)^{\beta}}}{1+(\theta-1)\left(1-e^{-(\alpha x)^{\beta}}\right)}\right] .
$$

Let $X$ be a random variable with density (5), then

and

$$
\bar{F}(x) E[h(x) \mid X \geq x]=\frac{e^{-(\alpha x)^{\beta}}}{\left[1+(\theta-1)\left[1-e^{-(\alpha x)^{\beta}}\right]\right]}
$$

and finally

$$
\bar{F}(x) E[g(x) \mid X \geq x]=\frac{e^{-(\alpha x)^{\beta}}\left[2 \theta-(\theta-1) e^{-(\alpha x)^{\beta}}\right]}{2 \theta\left[1+(\theta-1)\left[1-e^{-(\alpha x)^{\beta}}\right]\right]^{2}}
$$

$$
\begin{aligned}
& \eta(x) h(x)-g(x)=\frac{1}{2} h(x)\left[1-e^{-(\alpha x)^{\beta}}\right]^{a}, \\
& S^{\prime}(x)=\frac{\eta^{\prime}(x) h(x)}{\eta(x) h(x)-g(x)}=\frac{a \beta \alpha^{\beta} x^{\beta-1} e^{-(\alpha x)^{\beta}}}{\left[1-e^{-(\alpha x)^{\beta}}\right]} .
\end{aligned}
$$

Then, we have

$$
S(x)=\operatorname{aln}\left[1-e^{-(\alpha x)^{\beta}}\right] .
$$

Then, $X$ has the pdf (5).

Corollary: Let $X: \Omega \rightarrow(\theta, \infty)$ be a continuous random variable and let $h(x)$ be as in Proposition (1). Then the random variable $X$ has the pdf (5) if and only if the functions $g$ and $h$ defined in Theorem 1 satisfy the following differential equation

$$
\frac{\eta^{\prime}(x) h(x)}{\eta(x) h(x)-g(x)}=\frac{a \beta \alpha^{\beta} x^{\beta-1} e^{-(\alpha x)^{\beta}}}{\left[1-e^{-(\alpha x)^{\beta}}\right]}
$$

The general solution of the above differential equation is

$$
\eta(x)=\left[1-e^{-(\alpha x)^{\beta}}\right]^{a}\left\{-\int \frac{a \beta \alpha^{\beta} x^{\beta-1} e^{-(\alpha x)^{\beta}}}{\left[1-e^{-(\alpha x)^{\beta}}\right]} \times \frac{g(x)}{h(x)} d x+K\right\},
$$

where $K$ is a constant. There is a set of functions satisfying the differential equation (15) is given in Proposition 1 with $K=0$. Moreover, there are other triplets $(h, g, \eta)$ satisfying the conditions of Theorem 1 .

\section{Maximum Likelihood Estimation}

Several approaches for parameter estimation were proposed in the literature but the maximum likelihood method is the most commonly employed. The maximum likelihood estimators (MLEs) enjoy desirable properties and can be used when constructing confidence intervals and also in test statistics. The normal approximation for these estimators in large sample theory is easily handled either analytically or numerically. So, 
we consider the estimation of the unknown parameters for this family from complete samples only by maximum likelihood. Here, we determine the MLEs of the parameters of the new family of distributions from complete samples only. Let $x_{1}, \ldots, x_{n}$ be a random sample from the TG-W distribution with parameters $\lambda, \theta, \alpha$ and $\beta$. Let $\Theta=(\lambda, \theta, \alpha, \beta)^{\mathrm{T}}$ be the $(4 \times 1)$ parameter vector. Then, the log-likelihood function for $\Theta$, say $\ell=\ell(\Theta)$, is given by

$\ell=n \log \theta+n \log \beta+n \beta \log \alpha+(\beta-1) \sum_{i=0}^{n} \log \left(x_{i}\right)+\sum_{i=0}^{n} \log s_{i}-2 \sum_{i=0}^{n} \log z_{i}+$

$\sum_{i=0}^{n} \log p_{i}$,

where

and

$$
s_{i}=\mathrm{e}^{-\alpha^{\beta} x_{i}^{\beta}}=\mathrm{e}^{-\left(\alpha x_{i}\right)^{\beta}}, z_{i}=\left[1+(\theta-1)\left(1-s_{i}\right)\right]
$$

$$
p_{i}=\left[1+\lambda-\frac{2 \lambda \theta\left(1-s_{i}\right)}{z_{i}}\right]
$$

Equation (15) can be maximized either directly by using the R (optim function), SAS (PROC NLMIXED) or Ox program (sub-routine MaxBFGS) or by solving the nonlinear likelihood equations obtained by differentiating (15). The score vector components, say $\mathbf{U}(\Theta)=\frac{\partial \ell}{\partial \Theta}=\left(\frac{\partial \ell}{\partial \lambda}, \frac{\partial \ell}{\partial \theta}, \frac{\partial \ell}{\partial \alpha}, \frac{\partial \ell}{\partial \beta}\right)^{\mathrm{T}}=\left(U_{\lambda}, U_{\theta}, U_{\alpha}, U_{\beta}\right)^{\mathrm{T}}$, are given by

$$
\begin{aligned}
U_{\lambda}=\sum_{i=0}^{n} \frac{t_{i}}{p_{i}}, U_{\theta} & =\frac{n}{\theta}+-2 \sum_{i=0}^{n} \frac{1-s_{i}}{z_{i}}+\sum_{i=0}^{n} \frac{q_{i}}{p_{i}}, U_{\alpha} \\
= & \frac{n \beta}{\alpha}+\sum_{i=0}^{n} \frac{m_{i}}{s_{i}}+2(\theta-1) \sum_{i=0}^{n} \frac{m_{i}}{z_{i}}+\sum_{i=0}^{n} \frac{a_{i}}{p_{i}}
\end{aligned}
$$

and

$$
U_{\boldsymbol{\beta}}=\frac{n}{\beta}+n \log \alpha+\sum_{i=0}^{n} \log \left(x_{i}\right)+\sum_{i=0}^{n} \frac{w_{i}}{s_{i}}+2 \sum_{i=0}^{n} \frac{(\theta-1) w_{i}}{z_{i}}+\sum_{i=0}^{n} \frac{b_{i}}{p_{i}},
$$

where

$$
\begin{gathered}
m_{i}=\frac{-\beta \alpha^{\beta-1} x_{i}^{\beta}}{\mathrm{e}^{\left(\alpha x_{i}\right)^{\beta}}}, w_{i}=\frac{-\left(\alpha x_{i}\right)^{\beta} \log \left(\alpha x_{i}\right)}{\mathrm{e}^{\left(\alpha x_{i}\right)^{\beta}}}, b_{i}=\frac{-2 \lambda \theta\left[-w_{i} z_{i}+(\theta-1)\left(1-s_{i}\right) w_{i}\right]}{z_{i}^{2}}, \\
q_{i}=-\frac{2 \lambda\left(1-s_{i}\right)\left[z_{i}-\theta\left(1-s_{i}\right)\right]}{z_{i}^{2}}, a_{i}=\frac{-2 \lambda \theta\left[-m_{i} z_{i}+(\theta-1)\left(1-s_{i}\right) m_{i}\right]}{z_{i}^{2}} \text { and } t_{i} \\
=1-\frac{2 \theta\left(1-s_{i}\right)}{z_{i}} .
\end{gathered}
$$

Setting the nonlinear system of equations $U_{\lambda}=U_{\theta}=U_{\alpha}=U_{\beta}=\mathbf{0}$ and solving them simultaneously yields the MLE $\widehat{\Theta}=(\hat{\lambda}, \hat{\theta}, \hat{\alpha}, \hat{\beta})^{\mathrm{T}}$ of $\Theta=(\lambda, \theta, \alpha, \beta)^{\mathrm{T}}$. These equations cannot be solved analytically and statistical software can be used to solve them numerically using iterative methods such as the Newton-Raphson type algorithms. For interval estimation of the model parameters, we require the observed information matrix

$$
J(\Theta)=-\left(\begin{array}{cccc}
U_{\lambda \lambda} & U_{\lambda \theta} & U_{\lambda \alpha} & U_{\lambda \beta} \\
U_{\theta \lambda} & U_{\theta \theta} & U_{\theta \alpha} & U_{\theta \beta} \\
U_{\alpha \lambda} & U_{\alpha \theta} & U_{\alpha \alpha} & U_{\alpha \beta} \\
U_{\beta \lambda} & U_{\beta \theta} & U_{\beta \alpha} & U_{\beta \beta}
\end{array}\right),
$$


Under standard regularity conditions when $n \rightarrow \infty$, the distribution of $\widehat{\Theta}$ can be approximated by a multivariate normal $N_{4}\left(0, J(\widehat{\Theta})^{-1}\right)$ distribution to construct approximate confidence intervals for the parameters. Here, $J(\widehat{\Theta})$ is the total observed information matrix evaluated at $\widehat{\Theta}$. The method of the re-sampling bootstrap can be used for correcting the biases of the MLEs of the model parameters. Interval estimates may also be obtained using the bootstrap percentile method. Likelihood ratio tests can be performed for the proposed family of distributions in the usual way.

\section{Simulation Study}

In this section, a brief simulation study is conducted to examine the performance of the MLEs of TGW parameters. Inverse transform method is used to generate random observations from TGW distribution. We generate 1000 samples of size, $n=50,100,500$ and $n=1000$ of TGW distribution. The evaluation of estimates was based on the bias of the MLEs of the model parameters, the mean squared error (MSE) of the MLEs. The empirical study was conducted with software $\mathrm{R}$ and the results are given in Table 1 . The values in Table 1 indicate that the estimates are quite stable and, more importantly, are close to nominal values when $n$ goes to infinity. It is observed from Table 1 that the biases and MSEs decreases as $n$ increases. The simulation study shows that the maximum likelihood method is appropriate for estimating the parameters of TGW distribution. In fact, the MSEs of the parameters tend to be closer to the zero when $\mathrm{n}$ increases. This fact supports that the asymptotic normal distribution provides an adequate approximation to the finite sample distribution of the MLEs. The normal approximation can be improved by using bias adjustments to these estimators.

Table 1: Biases and MSEs for the MLEs of the parameters of the TGW distribution

\begin{tabular}{lllllllllll}
\hline $\begin{array}{c}\boldsymbol{\alpha}=\mathbf{0 . 5}, \\
\mathbf{0 . 5}\end{array}$ & $\boldsymbol{\beta}=$ & Bias & & & & MSE & & & \\
\hline $\boldsymbol{\theta}$ & $\boldsymbol{\lambda}$ & $\mathbf{n}$ & $\boldsymbol{\theta}$ & $\boldsymbol{\lambda}$ & $\boldsymbol{\alpha}$ & $\boldsymbol{\beta}$ & $\boldsymbol{\theta}$ & $\boldsymbol{\lambda}$ & $\boldsymbol{\alpha}$ & $\boldsymbol{\beta}$ \\
\hline $\mathbf{0 . 5}$ & $\mathbf{0 . 5}$ & $\mathbf{5 0}$ & 1.049 & 0.877 & 0.701 & 0.622 & 0.549 & 0.945 & 0.154 & 0.322 \\
& & $\mathbf{1 0 0}$ & 0.549 & 0.349 & 0.443 & 0.559 & 0.217 & 0.444 & 0.049 & 0.173 \\
& & $\mathbf{5 0 0}$ & 0.134 & 0.144 & 0.105 & 0.513 & 0.019 & 0.103 & 0.011 & 0.059 \\
& & $\mathbf{1 0 0 0}$ & 0.071 & 0.011 & 0.015 & 0.5 & 0.030 & 0.054 & 0.001 & 0.013 \\
\hline $\mathbf{2}$ & $\mathbf{0 . 5}$ & $\mathbf{5 0}$ & 0.933 & 0.649 & 0.504 & 0.632 & 0.249 & 0.547 & 0.094 & 0.103 \\
& & $\mathbf{1 0 0}$ & 0.519 & 0.394 & 0.301 & 0.496 & 0.103 & 0.343 & 0.043 & 0.048 \\
& & $\mathbf{5 0 0}$ & 0.104 & 0.104 & 0.148 & 0.338 & 0.018 & 0.109 & 0.011 & 0.014 \\
& & $\mathbf{1 0 0 0}$ & 0.034 & 0.047 & 0.039 & 0.079 & 0.002 & 0.049 & 0.005 & 0.002 \\
\hline $\mathbf{3}$ & $\mathbf{0 . 7}$ & $\mathbf{5 0}$ & 1.273 & 1.011 & 0.335 & 0.445 & 0.949 & 1.031 & 0.334 & 0.509 \\
& & $\mathbf{1 0 0}$ & 0.553 & 0.589 & 0.147 & 0.049 & 0.549 & 0.566 & 0.109 & 0.202 \\
& & $\mathbf{5 0 0}$ & 0.104 & 0.147 & 0.049 & 0.017 & 0.107 & 0.193 & 0.039 & 0.018 \\
& & $\mathbf{1 0 0 0}$ & 0.049 & 0.056 & 0.001 & 0.003 & 0.043 & 0.044 & 0.003 & 0.001 \\
\hline
\end{tabular}




\section{The log-transmuted geometric-Weibull (LTW-W) regression model}

The TGW distribution with four parameters $\alpha>0, \theta>0, a>0$ and $b>0$, introduced in Section 1. Let $X$ is a random variable following the TGW density function and $Y$ is defined by $Y=\log (X)$. The density function of $Y$ obtained by replacing $\beta=1 / \sigma$ and $\mu=-\log (\alpha)$ reduces to

$$
f(y)=\frac{\frac{\theta}{\sigma} \exp \left[\left(\frac{y-\mu}{\sigma}\right)-\exp \left(\frac{y-\mu}{\sigma}\right)\right]}{\left[1+(\theta-1)\left\{1-\exp \left[-\exp \left(\frac{y-\mu}{\sigma}\right)\right]\right\}\right]^{2}}\left[1+\lambda-\frac{2 \lambda\left\{1-\exp \left[-\exp \left(\frac{y-\mu}{\sigma}\right)\right]\right\}}{1+(\theta-1)\left\{1-\exp \left[-\exp \left(\frac{y-\mu}{\sigma}\right)\right]\right\}}\right]
$$

where $y \in \Re, \mu \in \Re, \sigma>0, \theta>0$ and $\lambda>0$. We refer to Equation (17) as the LTGW distribution, say $Y: \operatorname{LTGW}(\theta, \lambda, \sigma, \mu)$, where $\mu \in \mathfrak{R}$ is the location parameter, $\sigma>0$ is the scale parameter and $\theta$ and $\lambda$ are shape parameters.

The corresponding survival function is

$$
s(y)=1-\frac{\theta\left\{1-\exp \left[-\exp \left(\frac{y-\mu}{\sigma}\right)\right]\right\}}{1+(\theta-1)\left\{1-\exp \left[-\exp \left(\frac{y-\mu}{\sigma}\right)\right]\right\}}\left[1+\frac{\lambda \exp \left[-\exp \left(\frac{y-\mu}{\sigma}\right)\right]}{1+(\theta-1)\left\{1-\exp \left[-\exp \left(\frac{y-\mu}{\sigma}\right)\right]\right\}}\right]
$$

and the hrf is simply $h(y)=f(y) / S(y)$. The standardized random variable $Z=(Y-$ $\mu) / \sigma$ has density function

$$
f(z)=\frac{\theta \exp [z-\exp (z)]}{[1+(\theta-1)\{1-\exp [-\exp (z)]\}]^{2}}\left[1+\lambda-\frac{2 \lambda\{1-\exp [-\exp (z)]\}}{1+(\theta-1)\{1-\exp [-\exp (z)]\}}\right]
$$

Parametric regression models to estimate univariate survival functions for censored data are widely used. A parametric model that provides a good fit to lifetime data tends to yield more precise estimates of the quantities of interest. Based on the LTGW density, we propose a linear location-scale regression model linking the response variable $y_{i}$ and the explanatory variable vector $\mathbf{v}_{i}^{T}=\left(v_{i 1}, \ldots, v_{i p}\right)$ given by

$$
y_{i}=\mathbf{v}_{i}^{T} \boldsymbol{\beta}+\sigma z_{i}, \mathrm{i}=1, \ldots, \mathrm{n}
$$

where the random error $z_{i}$ has density function (19), $\boldsymbol{\beta}=\left(\beta_{1}, \ldots, \beta_{p}\right)^{T}, \sigma>0, \theta>0$ and $\lambda>0$ are unknown parameters. The parameter $\mu_{i}=\mathbf{v}_{i}^{T} \boldsymbol{\beta}$ is the location of $y_{i}$. The location parameter vector $\mu=\left(\mu_{1}, \ldots, \mu_{n}\right)^{T}$ is represented by a linear model $\mu=V \beta$, where $V=\left(v_{1}, \ldots, v_{n}\right)^{T}$ is a known model matrix.

Consider a sample $\left(y_{1}, v_{1}\right), \ldots,\left(y_{n}, v_{n}\right)$ of $n$ independent observations, where each random response is defined by $y_{i}=\min \left\{\log \left(x_{i}\right), \log \left(c_{i}\right)\right\}$. We assume non-informative censoring such that the observed lifetimes and censoring times are independent. Let $F$ and $C$ be the sets of individuals for which $y_{i}$ is the log-lifetime or log-censoring, respectively. The log-likelihood function for the vector of parameters $\tau=\left(\alpha, \lambda, \sigma, \beta^{T}\right)^{T}$ from model (20) has the form $l(\tau)=\sum_{i \in F} l_{i}(\tau)+\sum_{i \in C} l_{i}^{(c)}(\tau)$, where $l_{i}(\tau)=$ $\log \left[f\left(y_{i}\right)\right], l_{i}^{(c)}(\tau)=\log \left[S\left(y_{i}\right)\right], f\left(y_{i}\right)$ is the density (17) and $S\left(y_{i}\right)$ is the survival function (18) of $Y_{i}$. Then, the total $\log$-likelihood function for $\tau$ reduces to

$$
\begin{aligned}
& \ell(\tau)=r \log \left(\frac{\theta}{\sigma}\right)+\sum_{i \in F}\left(z_{i}-u_{i}\right)-\sum_{\in F} \log \left[1+(\theta-1)\left\{1-\exp \left[-u_{i}\right]\right\}\right]^{-2} \\
& +\sum_{\in F} \log \left[1+\lambda-\frac{2 \lambda\left\{1-\exp \left[-u_{i}\right]\right\}}{1+(\theta-1)\left\{1-\exp \left[-u_{i}\right]\right\}}\right] \\
& +\sum_{i \in C} \log \left\{1-\frac{\theta\left\{1-\exp \left[-u_{i}\right]\right\}}{1+(\theta-1)\left\{1-\exp \left[-u_{i}\right]\right\}}\left[1+\frac{\lambda \exp \left[-u_{i}\right]}{1+(\theta-1)\left\{1-\exp \left[-u_{i}\right]\right\}}\right]\right\}
\end{aligned}
$$


where $u_{i}=\exp \left(z_{i}\right), z_{i}=\left(y_{i}-v_{i}^{T} \beta\right) / \sigma$ and $r$ is the number of uncensored observations (failures) and $c$ is the number of the censored observations. The MLE $\hat{\tau}$ of the vector of unknown parameters can be evaluated by maximizing the log-likelihood (21). We use the statistical software $\mathrm{R}$ to determine the estimate $\hat{\tau}$.

Under standard regularity conditions, the asymptotic distribution of $(\hat{\tau}-\tau)$ is multivariate normal $N_{p+3}\left(0, K(\tau)^{-1}\right)$, where $K(\tau)$ is the expected information matrix. The asymptotic covariance matrix $K(\tau)^{-1}$ of $\hat{\tau}$ can be approximated by the inverse of the $(p+3) \times(p+3)$ observed information matrix $-(\tau)$. The elements of the observed information matrix $-\ddot{\mathrm{E}}(\tau)$, namely $-\mathrm{七}_{\theta \theta},-\mathrm{七}_{\theta \lambda}$, $-\mathrm{七}_{\theta \sigma},-\mathrm{七}_{\theta \beta_{j}},-\mathrm{七}_{\lambda \lambda},-\mathrm{七}_{\lambda \sigma},-\mathrm{七}_{\lambda \beta_{j}},-\mathrm{七}_{\sigma \sigma},-\mathrm{七}_{\sigma \beta_{j}}$ and $-\mathrm{七}_{\beta_{j} \beta_{s}}$ for $j, s=1, \ldots, p$, are evaluated numerically. The approximate multivariate normal distribution $N_{p+3}\left(0,-\ddot{€}(\tau)^{-1}\right)$ for $\hat{\tau}$ can be used in the classical way to construct approximate confidence regions for some parameters in $\tau$.

\section{Applications}

In this section, we provide an application to real data set to illustrate the flexibility of the TGW distribution. The parameters are estimated by maximum likelihood method and $\mathrm{R}$ statistical software is used for computations. First, we describe the data sets and then determine the MLEs (and the corresponding standard errors) of the parameters. In order to compare models with the proposed distribution, we apply goodness-of-fit tests to verify which distribution fits better the real data set. The statistics Cramer von Mises $\left(\mathrm{W}^{*}\right)$ and Anderson Darling $\left(\mathrm{A}^{*}\right)$ are described in details in Chen and Balakrishnan (1995). The log-likelihood values and Akaike Information Criterion (AIC) are also obtained for all models and used to decide best model. In general, the smaller the values of these statistics, the better the fit to the data.

We compare the performance of the TGW distribution with other well-known families given in Table 2 .

Table 2: Fitted families and their abbreviations

\begin{tabular}{ll}
\hline Families & References \\
\hline Weibull & \\
Odd Log-Logistic-Weibull (OLL-W) & da Cruz et al. (2014) \\
Kumaraswamy-Weibull (Kum-W) & Cordeiro and de Castro (2011) \\
Exponentiated Generalized-Weibull (EG-W) & Cordeiro et al. (2013) \\
Weibull-Weibull (W-W) & Bourguignon et al. (2014) \\
Beta-Weibull (B-W) & Eugene et al. (2002) \\
Transmuted Geometric-Weibull (TGW) & Proposed \\
\hline
\end{tabular}




\subsection{Strength of glass fibres}

The data set represents the strength of $1.5 \mathrm{~cm}$ glass fibres, measured at National physical laboratory, England (see, Smith and Naylor [46]). The data are: 0.55, 0.93, 1.25, 1.36, $1.49,1.52,1.58,1.61,1.64,1.68,1.73,1.81,2.00,0.74,1.04,1.27,1.39,1.49,1.53,1.59$, $1.61,1.66,1.68,1.76,1.82,2.01,0.77,1.11,1.28,1.42,1.50,1.54,1.60,1.62,1.66,1.69$, $1.76,1.84,2.24,0.81,1.13,1.29,1.48,1.50,1.55,1.61,1.62,1.66,1.70,1.77,1.84,0.84$, $1.24,1.30,1.48,1.51,1.55,1.61,1.63,1.67,1.70,1.78,1.89$.

Table 3 gives $\mathrm{W}^{*}$ and $\mathrm{A}^{*}$ statistics, AIC and log-likelihood values. Based on Tables 2, it is clear that TGW distribution provides the overall best fit and therefore could be chosen as the more adequate model from other models for explaining the used data set.

Table3: Parameter estimations of fitted distributions

\begin{tabular}{|c|c|c|c|c|c|c|c|c|}
\hline \multirow[t]{2}{*}{ Models } & \multicolumn{4}{|c|}{ Parameters } & \multirow[t]{2}{*}{$-\ell$} & \multirow[t]{2}{*}{ AIC } & \multirow[t]{2}{*}{$\mathbf{A}^{*}$} & \multirow[t]{2}{*}{$\mathbf{W}^{*}$} \\
\hline & $\boldsymbol{\theta}$ & $\lambda$ & $\alpha$ & $\beta$ & & & & \\
\hline \multirow[t]{2}{*}{$\mathbf{W}$} & & & 5.781 & 1.628 & 15.206 & 34.413 & 1.303 & 0.237 \\
\hline & & & 0.576 & 0.037 & & & & \\
\hline \multirow[t]{2}{*}{ OLL-W } & & 0.943 & 6.025 & 1.623 & 15.186 & 36.373 & 1.282 & 0.233 \\
\hline & & 0.267 & 1.340 & 0.043 & & & & \\
\hline \multirow[t]{2}{*}{ KUM-W } & 0.497 & 0.199 & 7.009 & 1.346 & 13.408 & 34.817 & 0.829 & 0.144 \\
\hline & 0.138 & 0.027 & 0.002 & 0.002 & & & & \\
\hline \multirow[t]{2}{*}{ EG-W } & 0.188 & 0.762 & 6.705 & 1.319 & 14.685 & 37.370 & 1.111 & 0.199 \\
\hline & 0.034 & 0.121 & 0.006 & 0.006 & & & & \\
\hline \multirow[t]{2}{*}{ W-W } & 0.052 & 3.041 & 1.089 & 1.299 & 14.429 & 36.858 & 1.109 & 0.199 \\
\hline & 0.111 & 2.061 & 0.559 & 0.623 & & & & \\
\hline \multirow[t]{2}{*}{ B-W } & 0.637 & 0.199 & 7.073 & 1.350 & 13.994 & 35.988 & 0.966 & 0.172 \\
\hline & 0.128 & 0.028 & 0.003 & 0.002 & & & & \\
\hline \multirow[t]{2}{*}{ TG-W } & 0.035 & 0.773 & 3.051 & 1.125 & 11.538 & 31.076 & 0.495 & 0.088 \\
\hline & 0.046 & 0.284 & 1.015 & 0.280 & & & & \\
\hline
\end{tabular}

More information can be provided in Figure 3 by a histogram of the data with fitted lines of the pdfs for all distributions. We present the plots of the fitted density, cumulative and survival functions with the probability-probability (P-P) plot for the TGW distribution in Figure 4. They reveal a good adjustment for the data of the estimated density, cumulative and survival functions of the TGW distribution. 

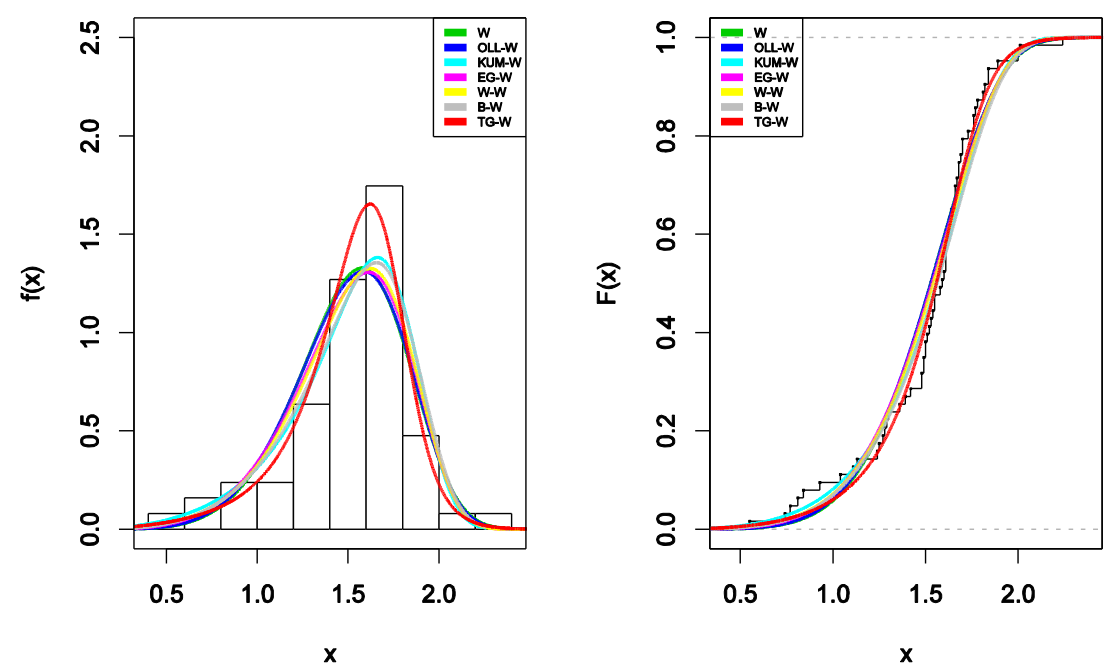

Figure 3: Fitted pdfs on histogram of first data set

Fitted Density

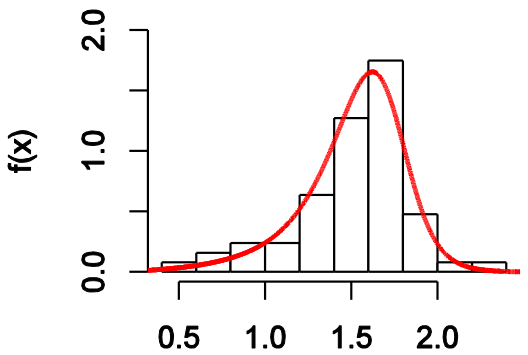

$\mathbf{X}$

Fitted Survival

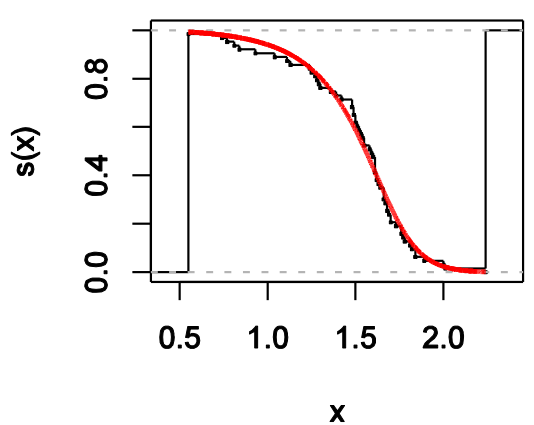

Fitted CDF

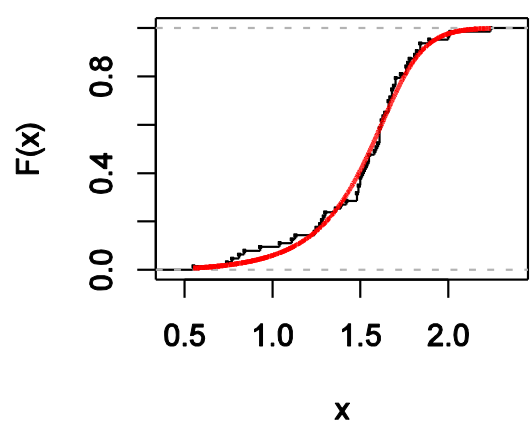

PP Plot

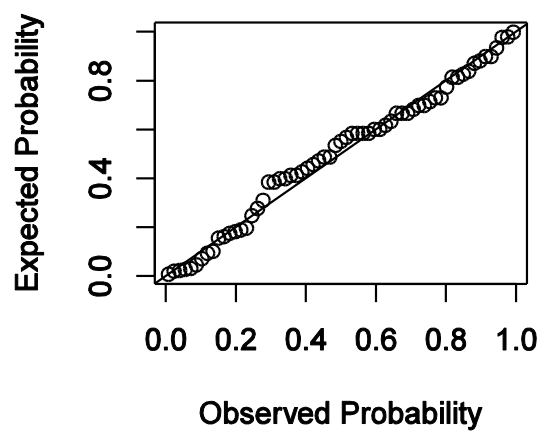

Figure 4: Fitted plots for TGW distribution 


\subsection{Multiply censored relay data}

The used data set represents the production relay and on a proposed design change $(n=$ 35). Engineering experience suggested that lifetime has a Weibull distribution. Engineering sought to compare the production and proposed designs over the range of test currents. These data are also reported and analyzed in Cordeiro et al. (2017). LTGW regression model is adopted to analyze these data set. The variables involved in the study are: $y_{i}$ - observed thounsands of cycles; cens $s_{i}$ - censoring indicator ( $0=$ censoring, $1=$ lifetime observed) and $x_{i 1}$ - production (16 amps, 26 amps, 28 amps). We consider the following regression model

$$
y_{i}=\beta_{1}+\beta_{2} v_{i}+\sigma z_{i}
$$

where $y_{i}$ has the LTGW density (17), for $i=1, \ldots, 35$. Table 4 lists the MLEs of the model parameters of the LTGW regression model fitted to the current data and the loglikelihood, AIC and BIC statistics. Based on the Table 4, it is clear that $\beta_{1}$ is statistically significant at the 5\% level and then there is a significant difference among the levels of the production for the thousands of cycles.

Table 4: MLEs of the parameters (standard errors in parentheses and $\boldsymbol{p}$-values in $[\cdot])$ and the log-likelihood, AIC and BIC measures.

\begin{tabular}{lllllllll}
\hline Model & $\boldsymbol{\theta}$ & $\boldsymbol{\lambda}$ & $\boldsymbol{\sigma}$ & $\boldsymbol{\beta}_{\mathbf{0}}$ & $\boldsymbol{\beta}_{\mathbf{1}}$ & $-\boldsymbol{\ell}$ & AIC & BIC \\
\hline LTGW & 4.834 & 0.001 & 0.3257 & 7.504 & -0.065 & 22.146 & 54.293 & 62.071 \\
& $(0.625)$ & $(0.522)$ & $(3.104)$ & $(0.757)$ & $(0.014)$ & & & \\
& & & & {$[<0.001]$} & {$[<0.001]$} & & & \\
\hline
\end{tabular}

The plots in Figure 5(a) provide the Kaplan-Meier (KM) estimate and the estimated survival functions of the LTGW regression model. In view of Figure 5(a), there is no significant differences between the 26 and 28 amps levels survival functions. The plots of the hrf in Figure 5(b) corresponding to the thousands of cycles variable under the LTGW regression model indicate that the hrf is larger for 16 amps level than for 26 and 28 amps levels. Based on these plots, we conclude that the LTGW regression model provides a good fit to this data.

(a)

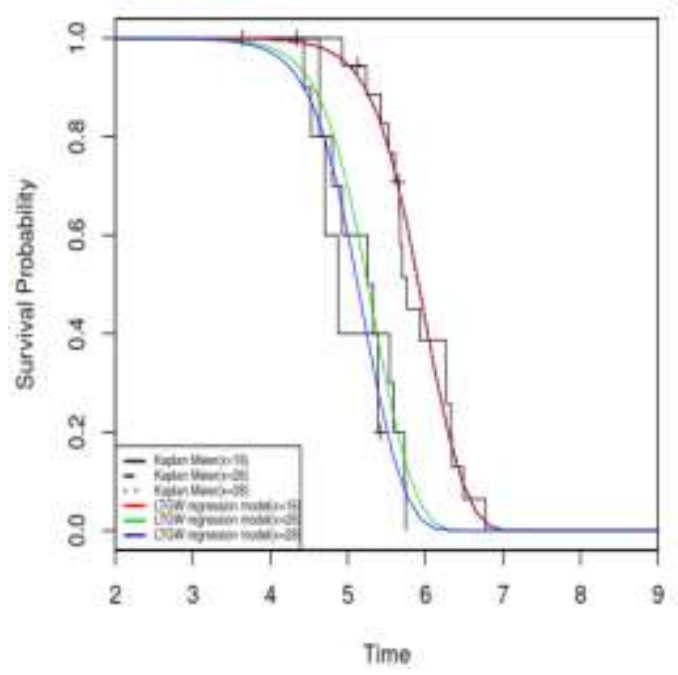

(b)

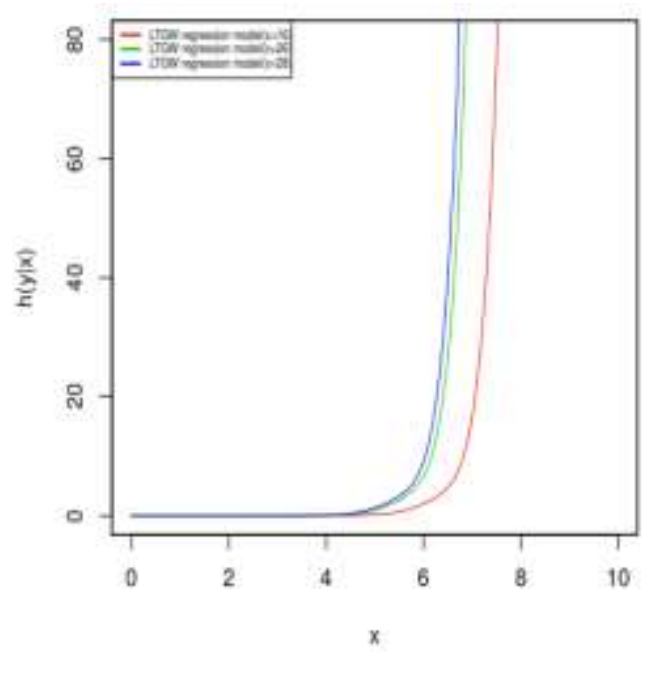


Figure 5: (a) Estimated survival functions and the empirical survival: LTGW regression model versus KM. (b) Fitted hrf using the LTGW regression model for the level production (16, 26 and 28 amps.).

\section{Conclusions}

We introduce the new lifetime distribution named the Transmuted Geometric-Weibull (TGW) distribution. Some of its mathematical properties are obtained. The maximum likelihood method is used to estimate the model parameters and the performance of the maximum likelihood estimators are discussed in terms of biases and mean squared errors. Two applications of the proposed family prove empirically its flexibility to model the real data sets. The log location-scale regression model based on a new generated distribution is introduced and discussed by means of real data application. Finally, it is clear that the proposed distribution provides better fits than other competitive models for used data sets.

\section{References}

1. Afify A. Z., Alizadeh, M., Yousof, H. M., Aryal, G. and Ahmad, M. (2016a). The transmuted geometric-G family of distributions: theory and applications. Pak. J. Statist., 32(2),139-160.

2. Afify, A. Z., Cordeiro, G. M., Yousof, H. M., Saboor, A., \& Ortega, E. M. M. (2016). The Marshall-Olkin additive Weibull distribution with variable shapes for the hazard rate. Hacettepe Journal of Mathematics and Statistics, Forthcoming.

3. Alzaatreh, A., Lee, C., \& Famoye, F. A. (2013). A new method for generating families of continuous distributions, Metron 71, 63-79.

4. Aryal, G. R., \& Tsokos, C. P. (2011). Transmuted Weibull distribution: a generalization of the Weibull probability distribution. European Journal of Pure and Applied Mathematics, 4, 89-102.

5. Aryal, G. R., \& Elbatal, I. (2015). On the Exponentiated Generalized Modified Weibull Distribution. Communications for Statistical Applications and Methods, 22, 333-348.

6. Aryal, G. R., Ortega, E. M., Hamedani, G. G. \& Yousof, H. M. The Topp-Leone Generated Weibull distribution: regression model, characterizations and applications, International Journal of Statistics and Probability, Forthcoming.

7. Balakrishanan, N., Leiva, V., Sanhuzea, A., \& Cabrera, E. (2009). Mixture inverse Gaussian distributions and its transformations, moments and applications. Statistics, 43, 91-104.

8. Chhikara, R. S., \& Folks, J. L. (1989). The inverse Gaussian distribution, Marcel Dekker, New York.

9. Cordeiro, G. M., Alizadeh, M., Ozel, G., Hosseini, B., Ortega, E. M. M., \& Altun, E. (2017). The generalized odd log-logistic family of distributions: properties, regression models and applications. Journal of Statistical Computation and Simulation, 87(5), 908-932. 
10. Cordeiro, G. M., Hashimoto, E. M., \& Ortega, E. M. M. (2014). The McDonald Weibull model. Statistics: A Journal of Theoretical and Applied Statistics, 48, 256-278.

11. Cordeiro, G. M., Ortega, E. M., \& da Cunha, D. C. C. (2013). The exponentiated generalized class of distributions.

12. Cordeiro, G. M., Ortega, E. M., \& Nadarajah, S. (2010). The Kumaraswamy Weibull distribution with application to failure data. Journal of the Franklin Institute, 347, 1399-1429.

13. Cordeiro, G. M., Ortega, E. M., \& Silva, G. O. (2012). The Kumaraswamy modified Weibull distribution: theory and applications. Journal of Statistical Computation and Simulation, 84,1387-1411.

14. da Silva, R., Thiago, A., Maciel, D., Campos, R., \& Cordeiro, G. (2013). A new lifetime model: the gamma extended Frechet distribution. Journal of Statistical Theory and Applications, 12, 39-54.

15. Efron, B. (1988). Logistic regression, survival analysis and the Kaplan-Meier curve. Journal of the American Statistical Association, 83, 414-425.

16. Elbatal, I., \& Aryal, G. (2013). On the transmuted additive Weibull distribution. Austrian Journal of Statistics, 42, 117--132.

17. Famoye, F., Lee, C., \& Olumolade, O. (2005). The Beta-Weibull Distribution, Journal of Statistical Theory and Applications, 4, 121-136.

18. Ghitany, M. E., Al-Hussaini, E. K., \& Al-Jarallah, R. A. (2005). Marshall-Olkin extended Weibull distribution and its application to censored data. Journal of Applied Statistics, 32, 1025-1034.

19. Hanook, S., Shahbaz, M. Q., Mohsin, M., \& Kibria, G. (2013). A Note on Beta Inverse Weibull Distribution, Communications in Statistics - Theory and Methods, 42, 320-335.

20. Khan, M. S., \& King, R. (2013). Transmuted modified Weibull distribution: a generalization of the modified Weibull probability distribution. European Journal of Pure and Applied Mathematics, 6, 66-88.

21. Lai, C. D., Xie, M., \& Murthy, D. N. P. (2001). Bathtub-shaped failure rate life distributions, Chapter 3, in Advances in Reliability, vol. 20 of Handbook of Statistics, pp. 69104.

22. Lai, C. D., Xie, M., \& Murthy, D. N. P. (2003). A modified Weibull distribution. IEEE Transactions on Reliability, 52, 33-37.

23. Lee, C., Famoye, F., \& Olumolade, O. (2007). Beta-Weibull distribution: some properties and applications to censored data. Journal of modern applied statistical methods, 6, 17.

24. Merovci, F. and Elbatal, I. (2013). The McDonald modified Weibull distribution: properties and applications. arXiv preprint arXiv:1309.2961.

25. Mudholkar, G. S., \& Srivastava, D. K. (1993). Exponentiated Weibull family for analyzing bathtub failure-real data. IEEE Transactions on Reliability, 42, 299302. 
26. Mudholkar, G. S., Srivastava, D. K., \& Freimer, M. (1995). The exponentiated Weibull family: a reanalysis of the busmotor-failure data. Technometrics, 37, 436-445.

27. Mudholkar, G. S., Srivastava, D. K., \& Kollia, G. D. (1996). A generalization of the Weibull distribution with application to the analysis of survival data. Journal of the American Statistical Association, 91, 1575-1583.

28. Nofal, Z. M., Afify, A. Z., Yousof, H. M., \& Cordeiro, G. M. (2017). The generalized transmuted-G family of distributions. Communications in StatisticsTheory and Methods, 46:8,4119-4136.

29. Nofal, Z. M., Afify, A. Z., Yousof, H. M., \& Louzada, F.(2016). Transmuted exponentiated additive Weibull distribution: properties and applications, Forthcoming.

30. Nofal, Z. M., Afify, A. Z., Yousof, H. M., Granzotto, D. C. T., \& Louzada, F. (2016). Kumaraswamy transmuted exponentiated additive Weibull distribution. International Journal of Statistics and Probability, 5, 78-99.

31. Ortega, E. M. M., Cordeiro, G. M., \& Hashimoto, E. M. (2011). A log-linear regression model for the Beta-Weibull distribution. Communications in StatisticsSimulation and Computations, 40, 1206-1235.

32. Pinho, L. G., Cordeiro, G. M., \& Nobre, J. S. (2015). The Harris extended exponential distribution. Communications in Statistics- Theory and methods, 44, 3486-3502.

33. Provost, S. B., Saboor, A., \& Ahmad, M. (2011). The gamma-Weibull distribution, Pak. Journal Stat., 27, 111--131.

34. Rezaei, S., Sadr, B. B., Alizadeh, M., \& Nadarajah, S. (2016). Topp-Leone generated family of distributions: Properties and applications. Communications in Statistics- Theory and Methods, Forthcoming.

35. Sarhan, A. M., \& Zaindin, M. (2009). Modified Weibull distribution, Applied Sciences, 11, 123-136.

36. Shahbaz, M. G., Shahbaz, S., \& Butt, N. M. (2102). The Kumaraswamy-inverse Weibull distribution. Pak. Journal Stat. Oper. Res., 8, 479-489.

37. Silva, G. O., Ortega, E. M. M., \& Cordeiro, G. M. (2010). The beta modified Weibull distribution. Lifetime Data Analysis, 16, 409-430.

38. Xie, M., Tang, Y., \& Goh, T. N. (2002). A modified Weibull extension with bathtub failure rate function. Reliability Engineering and System Safety, 76, 279285.

39. Xie, M. \& Lai, C. D. (1995). Reliability analysis using an additive Weibull model with bathtub-shaped failure rate function. Reliability Engineering and System Safety, 52, 87-93.

40. Yousof, H. M., Afify, A. Z., Alizadeh, M., Butt, N. S., Hamedani, G. G., \& Ali, M. M. (2015). The transmuted exponentiated generalized-G family of distributions. Pak. J. Stat. Oper. Res., 11, 441-464. 